\title{
Attention Deficit in Children With Attention Deficit Hyperactivity Disorder at Primary School Age Measured With the Attention Network Test (Ant): A Protocol for a Systematic Review and Meta-analysis
}

\section{Gabriela Oliwia Gradys ( $\sim$ gabriela.gradys@gmail.com )}

University of Gdansk Faculty of Social Sciences: Uniwersytet Gdanski Wydzial Nauk Spolecznych https://orcid.org/0000-0002-7206-8982

Małgorzata Lipowska

University of Gdansk Faculty of Social Sciences: Uniwersytet Gdanski Wydzial Nauk Spolecznych Łucja Bieleninik

University of Gdansk Faculty of Social Sciences: Uniwersytet Gdanski Wydzial Nauk Spolecznych

\section{Protocol}

Keywords: ADHD, attention deficit hyperactivity disorder, attention network task, ANT

Posted Date: July 12th, 2021

DOl: https://doi.org/10.21203/rs.3.rs-688069/v1

License: (a) (1) This work is licensed under a Creative Commons Attribution 4.0 International License.

Read Full License 


\section{Abstract}

\section{Background}

Attentional deficits are among the most bothersome symptoms in attention deficit hyperactivity disorder (ADHD). To date, the neurological basis of attentional deficits has not been fully described according to the diagnostic criteria. ADHD may result from deficits in various attributes of attention. There is no specialist neuropsychological diagnostic method that allows reliable distinction between primary attention disorders in the ethology of ADHD and secondary problems that may arise due to comorbidities. This protocol aims to systematically review the literature to evaluate patterns of attention common to school-age children either diagnosed with ADHD or at high risk of ADHD, as measured by the neuropsychological attention network test (ANT).

Methods

Our searchstrategy will consist of electronic databases (PubMed, PsychInfo, Web of Science, EMBASE, and Cochrane Library) and hand searching. Both prospective cohort studies and prospective studies of intervention effects will be included, provided they used the ANT. The primary output variable will be attention deficits. Screening and eligibility will be done independently by two reviewers based on prespecified eligibility criteria. Data extraction will be based on a pre-pilot data extraction form, and conducted by two authors independently. Study quality will be assessed by two authors independently. Any discrepancies identified at any stage of the review will be resolved by discussion or/and consultation with another reviewer. We plan a descriptive summary of the findings and a meta-analysis if the data allow.

\section{Discussion}

The research will identify patterns of neuropsychological ANT results characteristic to both school-age children diagnosed with ADHD and those at high risk of having ADHD. Our results could be used to check whether the pattern of a child's performance in the ANT corresponds to the characteristic pattern of the results of children with ADHD. At present, the ANT is used only in research ; the results of this review will serve as a useful benchmark. Hopefully, in the future, it will be possible to use the ANT in the wider diagnosis of ADHD.

Systematic review registration

PROSPERO: CRD42021249768

\section{Background}

Attention-deficit hyperactivity disorder (ADHD) is a neurodevelopmental disorder, including attention deficit disorder (ADD) and/or hyperactivity and impulsiveness (1). It is one of the most common neurodevelopmental disorders occurring in school-aged children, typically being noticed between six and 
nine years of age, and symptoms may continue into adulthood (2). The prevalence of ADHD is estimated to be between 6.7 and $7.8 \%$ (3). Consequently, in a typical class of 25-30 children, between 1 and 3 pupils may have an ADHD symptoms (4). Pupils with ADHD typically underachieve academically relative to non-ADHD classmates (5). Children and adolescents with ADHD are more likely to repeat a grade, to be referred for special education services, to be suspended from school, and to drop out of school relative to students without disabilities (6). These studies, as mentioned above, indicate that well-functioning attention seems to be a prerequisite for achieving good school results.

For those diagnosed with ADHD, attention deficits are among the symptoms that have the most negative impacts on their everyday lives. The neurological basis for the occurence of ADHD has not yet been fully described (7). The diagnostic criteria proposed by the American Psychological Association (1) are written in such a way that suggests that ADHD may result from deficits of various attention attributes. No neuropsychological methods exist in specialist diagnostics that allow reliable distinctions to be made between primary attention disorders in ADHD and secondary problems that may arise due to other disorders, such as specific learning disorder or behavioural disorders like conduct disorder (CD) and oppositional defiant disorder (ODD) (8-10). Primary attention deficits are caused by abnormal brain development, like in ADHD. Secondary attention deficits can appear during the lifespan as a result of unhealthy habits, such as suboptimal nutrition (11), loong screen time or poor sleeping; other neurodevelopmental and psychiatric disorders $(12,13)$, challenging life experiences $(14,15)$, and brain injury (16). The distinction between primary and secondary attention deficits is fundamental with respect to selecting relevant therapeutic approaches, pharmacological treatments, or combinations of these.At present, few clinical measurement tools exist to diagnose attention deficits in children (e.g., Continuous Performance Test, Child Behavior Checklist), and there are no methods available for diagnosis of primary attention deficit. The neuropsychological attention network test (ANT) may be useful in this capacity, but efforts are needed to investigate the performance of the test more carefully (17).

\section{Description of the method}

The ANT is a neuropsychological test based on attention network theory (18) which describes an attention system as comprising three networks:

1. Orienting network - relating to the ability to maintain increased sensitivity to new, upcoming stimuli.

2. Alerting network - concerning the ability to select stimuli and focus attention on the person's stimulus of interest.

3. Conflict or executive network - reflecting the ability to control a behavioural response in response to a stimulus that enables two alternative responses.

The ANT is a computer-based graphical tool that can be used to measure the efficiency of each of these networks by collecting information on the degree of correctness and the reaction time of participant responses to presented stimuli. In the test, participants are given various visual cues and/or warning tones while they attempt to indicate the direction in which a target arrow is pointing by pressing arrow 
keys on a keyboard. Participants are shown one of three types of flankers (neutral, compliant, and inconsistent) preceded by one of four types of clues (none, double, centre, and spatial). During the whole test, participants are shown a small cross at the centre of the screen. There are instructed to look at them during all the time. This is a fixation point, used mostly between the clue and the flanker, which makes their eyes relatively stationary. Thanks to that, they achieve retinal stabilization, fantks to that presented figure (clue or flanker) is tended to rapidly fade away. -Studies can be conducted using different rules, e.g., in the presentation time of the fixation, signal, target, and final fixation slides. The target presented can be fish (in the child version) rather than an arrow (in the adult version). Figure 1 illustrates the test. The numbers under each slide are a common presentation range. In the original ANT procedure (19), the each slide was shown for a random amount of time between 400 and $1600 \mathrm{msec}$ for the first fixation, $100 \mathrm{msec}$ for a cue, $400 \mathrm{msec}$ for fixation of a cue, $<1700 \mathrm{msec}$ for a target and $3500 \mathrm{msec}$ for the last fixation minus first fixation period and reaction time for a target. In the child version of ANT, times have been changed to adapt the original ANT (19), to children's abilities - slide timings were similar for first fixation slides and target, $150 \mathrm{msec}$ for a cue, $450 \mathrm{msec}$ for cue's fixation, $2000 \mathrm{msec}$ of feedback and $1000 \mathrm{msec}$ for finish fixation (20). Importantly, several versions of the ANT have been developed over the past two decades (for example: LANT (21), ANT-R (22), ANT-C

\section{Previous research and the rationale for the review}

To the best of our knowledge, little research has been done on measurement of attention deficit in children with ADHD using the ANT. Attention deficits in children with ADHD do not necessarily affect the entire spectrum of attention. They may only relate to specific features of attention. Through careful examination of deficits in the three attention networks, it is possible to identify exactly which areas of attention are impaired in children with ADHD and thus allow for the implementation of more effective treatment. To date, a single narrative review paper (17) and a single meta-analysis paper (26) have been undertaken on this topic. However, neither paper followed the PRISMA Statement (27). The narrative review by Vázquez-Marrufo, García-Valdecasas Colell (17) is based on 15 studies of changes in attention network measured with the ANT in school-age children and adults with ADD. The authors reported that the results of those studies were inconsistent. Some of the study results reported lower efficiency in all three attention networks in ADD children, but most of them found evidence for differences only in the alerting and control networks. Some of including research did not find any differences between the control and ADD groups. That may be coused by different the ANT procedures or analytical methods. In in a very recent publication on the ANT, Arora, Lawrence and Klein (26) developed an ANT database containing all studies that used the ANT as of 2019 and conducted two meta-analyses to explore task performance in children with and without ADHD. The authors of that paper found a significant difference between executive and alerting networks between children with and without ADHD. However, there were limitations in their methodology. Firstly, children with ADHD diagnosis and those exhibiting a high-risk of ADHD were not analysed separately, but combined into one group. In addition, their meta-analysis did not encompass all possible results of the ANT as analyses only incorporated overall reaction time and attention networks. The number of mistakes made by participants was not considered; thus, some questions still need to be answered. Importantly, the authors included only those studies that followed the 
original ANT method (19), which strongly impacted the results obtained. Another major source of uncertainty is that bias of included studies was not formally assessed, hindering the degree to which overall findings could be critically evaluated. Overall, there is abundant room for further analyses regarding the ANT in children with ADHD. Notably, It is not known with certainty whether a child with ADHD performs the ANT differently from a child without ADHD, and if so, in which outcomes these differences would manifest themselves, and in which direction any changes would be observed.

\section{Aim}

In this review, we aim to establish a single, distinctive pattern for ADHD children to perform the ANT, including the efficiency of attention network, reaction time, and numbers of all types of errors, none of which have been evaluated so far. This will allow comaprision of ANT results obtained in future experimental stuies are consistent with the pattern observed in ADHD respondents, or whether they are the result of hetermogeneity of the study group. This knowledge will allow the use of the ANT in further research and perhaps serve as a basis for the test's later standardization, which could make the ANT useable for broad diagnoses of attention. The last motivation to conduct the current systematic review was to provide an updated analysis of reaction time and attention networks, because the only metaanalysis which has been published included papers as of 2019 (26). In summary, we identified a need for rigorous systematic review with meta-analysis of the ANT to evaluate patterns of attention deficit characteristic of school-age children diagnosed with ADHD and those at high risk of ADHD.

This systematic review seeks to address the research question: Do primary school-age children diagnosed with ADHD or at risk of ADHD demonstrate differences in the efficiency of functioning of the three attention networks measured with the ANT when compared to non-ADHD children? In answering this question, we will refer to differences in performance of children diagnosed with ADHD and at risk of $A D H D$ in terms of alarm, conflict, and orientation networks compared to children without any ADHD symptoms. The following secondary questions were posited:

1. Do primary school-age children diagnosed with ADHD or at risk of ADHD compared to non-ADHD children demonstrate differences in correctness scores (number of commission and omission) measured with the ANT?

2. Do primary school-age children diagnosed with ADHD or at risk of ADHD compared to non-ADHD children demonstrate differences in the number of mistakes made in a particular type of clue or flankers measured with the ANT?

3. Do primary school-age children diagnosed with ADHD or at risk of ADHD compared to non-ADHD children demonstrate differences in the ratio times in a particular type of clue or flankers measured with the ANT?

Do primary school-age children diagnosed with ADHD or at risk of ADHD compared to non-ADHD children demonstrate differences in results of the ANT depending on the used version of ANT, e.g. LANT (21), ANTR (22), ANT-C (23), ANT-I (24), ANTI-V (25)? 


\section{Methods}

We undertook a comprehensive literature search following guidelines outlined in the Preferred reporting items for systematic review and meta-analysis protocols (PRISMA-P) (28). Our protocol was registered with the International Prospective Register of Systematic Reviews (PROSPERO) database (registration number: CRD42021249768).

\section{Eligibility Criteria}

Studies will be selected according to the criteria outlined below.

Participants: We will include studies of individuals of primary school age, both sexes without restriction to nationality, who had an ADHD diagnosis or were considered to be at high risk of ADHD. ADHD should have been diagnosed by a specialist (psychiatrist, clinical psychologist or any other qualification medical staff) based on DSM-5 (1) or based on either earlier versions of the DSM or the International Classification of Diseases (ICD) (29). The high-risk group of ADHD includes children with symptoms of ADHD measured by ADHD symptoms questionnaires (like CONNERS-3, Structured Diagnostic Interview Questionnaire for ADHD - or foreign equivalents).

Intervention/Exposure: The review will include studies regardless of the type of intervention for which the effectiveness was measured using the ANT.

Comparator/Control: We will include studies including individuals of primary school age, both sexes, without restriction to nationality, without ADHD symptoms or ADHD diagnosis.

Outcomes: Attention deficit measured by any version of the ANT, e.g. LANT (21), ANT-R (22), ANT-C (23), ANT-I (24), ANTI-V (25).

Primary outcomes: Performance index of the three attention networks (mean and standard deviation or median and range) of the ANT.

Secondary outcomes: Reaction time depending on the type of flanker and cue (mean and standard deviation or median and range). A number of omissions (missing answers) and a number of commissions (wrong answers) errors by flanker and cue type (correctness rate, the total number of mistakes made, or mean).

Study Type: We will include prospective cohort studies addressing attention deficit measured with the ANT and prospective studies of intervention effects with a control group (both randomized and nonrandomized controlled).

Location: We do not impose any restrictions on the area of the conducted research. 


\section{Search Strategy}

Information sources: The following electronic databases will be searched: PubMed, Psychlnfo, Web of Science, EMBASE, DARE, and the Cochrane Library. Electronic database searching will be supplemented by hand-searching reference lists of the included review articles to identify any additional studies. The search will be not restricted to any language (provided an English language translation of the abstract is available), sample size or year of publication. We will exclude editorials, letters, case studies and case series.

Search: Literature search strategies will be developed using Medical Subject Headings (30) or equivalent and text word terms and words related to the nosological unit and the ANT. In addition, Boolean operators along with proximity operators (parentheses and quotations) for each database will be applied. The search strategy included terms relating to condition (attention deficit disorder with hyperactivity [MeSH] OR ADHD) and measurement tool (Attentional Network Test" OR "Attentional Networks Test" OR "Attentional Network Task" OR "Attentional Networks task" OR "Attention Network Test" OR "Attention Networks Test" OR "Attention Network Task" OR "Attention Networks task"). We have already piloted the initial search strategy (including searching terms and filters) for PubMed in March 2021 to investigate whether the searching strategy allows us to find potentially relevant reports [see Additional file 1]. The pretested searching allowed us also to improve the search terms.Data Collection and analysis

\section{Study selection:}

One reviewer will search databases and handsearch the reference list of the included review articles. All potentially relevant records will be extracted to EndNote reference management software (31). At this stage, duplicates will be detected and deleted. Two review authors will screen titles and abstracts for their eligibility for inclusion under the above-defined criteria providing the reason(s) for rejection. Any discrepancies at the screening stage will be resolved by discussion with another reviewer. The eligibility criteria will be pre-tested on a reasonable sample of reports. We will obtain full reports for all titles that appear to meet the inclusion criteria or where there is any uncertainty. Two review authors independently will then screen the full-text reports and decide whether these meet the inclusion criteria proving the reason for rejection. Assessment of the relevance of studies will be conducted by clinical psychology researchers expert in the content area. They will seek additional information from each study's corresponding authors whenever it is necessary to resolve questions on eligibility. Eligibility criteria for each study will be assessed in order of importance, starting from participants, followed by the outcome, intervention/exposure, comparator/control and study design. Applying this strategy causes the first 'no' response to be the primary reason for excluding the study, and the remaining criteria will not be assessed. We will record the reasons for excluding studies. We will use a formal measure of an agreement to describe the extent to which assessments by two authors are the same. Disagreements at the assessment eligibility stage will be resolved by discussion with another reviewer. Multiple reports of the same study will be merged based on matching of the following study characteristics: author names, location and setting, numbers of participants and baseline data, and duration of the study. Where any 
uncertainties remain we will contact the corresponding authors. Neither of the review authors will be blind to the journal titles, or the study authors, or their institutions. The PRISMA template (32) will produce a flow chart showing details of studies included and excluded at each stage of the study selection process.

\section{Data extraction and management:}

Two authors independently will extract data from the studies based on a specifically designed and prepiloted data extraction form. Data extraction will be done by content area experts from the clinical psychology field who are familiar with the ANT, and who will be trained in how to code entries in the data collection form. Discrepancies will be resolved by discussion or/and consultation with another reviewer when needed. In case of discrepancies that cannot be resolved, we will contact the study authors; however, if this is unsuccessful, the discrepancies will be reported in the review. Corresponding authors will also be contacted to obtain any missing data. In case of multiple reports of the same study/project, we will extract data from each report separately and combine information across multiple data collection forms afterwards.

The following information will be extracted from the studies:

- Demographic characteristics of the population - age, sex, country of origin

- Clinical characteristics of the population- ADHD intensity evaluated by the results of the questionnaire (e.g., Conners 3); the sub-type of ADHD diagnosis; information by which the diagnosis was made (children who were assessed by a specialist will be allocated to the ADHD group; those whose assessment was based on questionnaire or interview will be assigned to the group of being at high risk of $A D H D$.)

- The ANT results - reaction times or their means/medians, number of errors or correctness index both variables, considering the division into Clue Type and Flanker type and the three attention networks results. In observational studies with repeated measurement or intervention studies with several time points, we will always extract baseline data. Description of performance in the ANT, the version of the ANT used, how the training of the ANT was performed, how the instructions were presented, the person conducting the test and their interventions with the child during the test, and any other data about the ANT.

- Characteristics of the interventions -Data on the types of intervention, their frequency, and their duration will be collected.

\section{Assessment from the risk of bias in included studies:}

An assessment of potential bias will be done independently by two review authors. Any discrepancies will be resolved by arbitration among reviewers, together with content area experts from clinical trial methodology if needed. To assess the risk of bias in each included study, we will use the Revised Cochrane Collaboration Risk of Bias Tool (RoB 2) for randomized trials (33) and the Risk Of Bias In Nonrandomized Studies - of Interventions (ROBINS-I) tool for non-randomized studies (34). The risk of bias will be judged as high, low, or unclear risk bias. For cohort studies, we will use The Newcastle-Ottawa 
Scale (35), which assess the quality of cohort studies by a judgement of the selection of the study groups, the comparability of these groups, and the ascertainment of either the exposure or outcome of interest (36).

\section{Data synthesis:}

We will provide a narrative synthesis of the findings from the included studies, structured around the participants (demographic and clinical characteristics), the ANT results and characteristics of the interventions (in the case of intervention-based research), along with a comparative table. Continuous data will be combined only if means and standard deviations are available and calculable. Provided there is no clear evidence of skewness in the distributions, the findings will be summarised using standard meta-analytical techniques described in the Cochrane Handbook for Systematic Reviews of Interventions (37).

\section{Data analysis:}

Data will be analyzed using the R programming language. (38)Three factors will be considered:

- Correctness - measured by the average number of errors made by children or the correctness rate.

- Reaction time - measured by the average number of reaction times to all stimuli or broken down by type of pointer, flanker, or type of clue.

- Attention network efficiency - measured by the difference in mean or median reaction times between:

1. Double clue vs no clue or tone vs no tone - for alerting network

2. Valid cue vs invalid clue - for orienting network

3. Congruent vs incongruent trial type - for executive network.

The three factors mentioned above will be compared between the ADHD clinical group and the control group as well as between the high-risk ADHD group and the control group to detect potential specific differences in children with ADHD to establish a characteristic pattern. We will conduct multiple metaanalyses, one for each version of the ANT.

\section{Subgroup analyses:}

- ADHD diagnosis vs high-risk of ADHD vs non-ADHD child - we will measure differences in the efficiency of functioning of the three attention networks, correctness scores (number of commission and omission), the number of mistakes and reaction time made in a particular type of clue or flankers measured with the ANT. It will be helpful to describe a characteristic pattern of attention disorder in ADHD and get an answer for the first, second and third question.

- We will compare all results obtained with all versions of the ANT with one another to answer the fourth secondary question. We will check whether the version of the ANT used affects the results obtained by all children. Furthermore, we will check whetherr they achieve similar effects, due to the 
fact that not every version of the ANT was designed to be used on children, but they are commonly used in that way. We will then be able to exclude these versions of the ANT which introduced bias.

- We will compare type A ADHD vs type B ADHD to check whether results of the ANT are different for each ADHD type. If such a difference were identified, it would be possible to predict the ADHD type based on the ANT results, and to describe the pattern of the ANT results for every type of ADHD.

We will compare symptoms of ADHD at intensity $A$ against symptoms at intensity $B$ to check whether the severity of the symptoms of attention disorders is reflected the results of the ANT. Were this found to be the case, it would be possible to predict attention disorders and their severity based on the results of the ANT. Children with ADHD diagnoses are a heterogeneousgroup with different intensities of attention deficit. Even in the DSM 5 diagnostic criteria, a child should obtain a minimum of 6 out of 9 criteria of inattention to gain ADHD diagnosis. That analysis could show that children with ADHD could also have slightly different results in the ANT, but still, it would be possible to differentiate them from children without attention deficits.

\section{Discussion}

We anticipate that our review will help future researchers using the ANT to find a reliable benchmark for observed results, and that it will contribute to furthering research into the possibility of using the ANT for a more comprehensive diagnosis of attention disorders in children, including ADHD. Perhaps the characteristic patterns of the ANT performed by children with ADHD we observe will be used as a reference point in comparative subjects to determine whether a given intervention (psychological, pharmacological, or other) used to reduce attention deficits in children is effective and results in actual, statistically significant differences in the efficiency of the attention networks of these children.

\section{Abbreviations}

ADD - Attention Deficit Disorder

ADHD - Attention-deficit/hyperactivity disorder

ANT - Attention Network Test

APA - American Psychological Association

CD - Conduct Disorder

ICD - International Classification of Diseases

MeSH - Medical Subject Headings

NOS - The Newcastle-Ottawa Scale 
ODD - Oppositional Defiant Disorder

PRISMA - Preferred Reporting Items for Systematic Reviews and Meta-Analysis

Rob 2 - Revised Cochrane Collaboration Risk of Bias Tool

ROBINS-I - Risk of Bias in Non-randomized Studies - of Interventions

\section{Declarations}

\section{Ethics approval and consent to participate:}

Not applicable

\section{Consent for publication}

Not applicable

\section{Availability of data and materials}

The main data generated and analyzed during this study will be included in the Systematic Review article. The additional datasets with detailed data used and analyzed during the current study will be available from the corresponding author on request.

\section{Competing interests}

The authors declare that they have no competing interests.

\section{Funding}

The "NeuroSmog: Determining the impact of air pollution on the developing brain" project is carried out within the TEAM-NET program of the Foundation for Polish Science co-financed by the European Union under the European Regional Development Fund (Nr. POIR.04.04.00-1763); Statutory funds of the Institute of Psychology of University of Gdańsk. The funding body does not influence the design of the study and the writing of the manuscript.

\section{Authors' contributions}

All authors were responsible and accountable for all part of the works related to the protocol. More specifically, GG and ML had the original idea. GG and $\measuredangle B$ contributed to the conception and design of the 
protocol. GG performed the first literature searches. GG and ŁB contributed to writing the manuscript. All authors revised the manuscript and approved the final version to be published.

\section{Acknowledgements}

We would like to thank James Grellier for reviewing our manuscript for linguistic correctness.[GJ1]

\section{Authors' information}

Dr hab. ML is a clinical psychologist, professor at the University of Gdańsk and the Child Psychology Team leader in the NeuroSmog project.

$\mathrm{PhD}$ of Psychology, ŁB currently works as an assistant professor at the University of Gdańsk in Poland and senior researcher at NORCE in Norway. She has conductded a total of six systematic reviews previously, including meta-analyses, so acts as the leading systematic review expert in that project.

GG is a graduate student at the University of Gdańsk and a scholarship holder in the child psychology team in the NeuroSmog project at the Institute of Applied Psychology at the Jagiellonian University. She was responsible for the programmed development and adaptation of the ANT in the Child version in NeuroSmog. She is in the process of analyzing data from the ANT.

\section{References}

1. APA. Diagnostic and Statistical Manual of Mental Disorders, 5th edition. In: Association AP, editor. 5th ed2013.

2. Roberts W, Milich R, Barkley RA. Primary symptoms, diagnostic criteria, subtyping, and prevalence of ADHD. In: Barkley RA, editor. Attention-deficit hyperactivity disorder: A handbook for diagnosis and treatment, 4th ed. New York, NY, US: Guilford Press; 2015. p. 51-80.

3. Thomas R, Sanders S, Doust J, Beller E, Glasziou P. Prevalence of attention-deficit/hyperactivity disorder: a systematic review and meta-analysis. Pediatrics. 2015;135(4):e994-1001.

4. DuPaul GJ, Reid R, Anastopoulos AD, Power TJ. Assessing ADHD symptomatic behaviors and functional impairment in school settings: Impact of student and teacher characteristics. School Psychology Quarterly. 2014;29(4):409-21.

5. Daley D, Birchwood J. ADHD and academic performance: Why does ADHD impact on academic performance and what can be done to support ADHD children in the classroom? Child: care, health and development. 2010;36:455-64. 
6. Siqueira CM, Gurgel-Giannetti J. [Poor school performance: an updated review]. Revista da Associacao Medica Brasileira (1992). 2011;57(1):78-87.

7. Weyandt LL, Gudmundsdottir BG. Developmental and Neuropsychological Deficits in Children with ADHD. In: Barkley RA, editor. Attention-deficit hyperactivity disorder: A handbook for diagnosis and treatment, 4th ed. New York, NY, US: Guilford Press; 2015. p. 116-39.

8. Pritchard AE, Nigro CA, Jacobson LA, Mahone EM. The Role of Neuropsychological Assessment in the Functional Outcomes of Children with ADHD. Neuropsychology Review. 2012;22(1):54-68.

9. Barkley RA. Psychological assessment of children with ADHD. In: Barkley RA, editor. Attentiondeficit hyperactivity disorder: A handbook for diagnosis and treatment, 4th ed. New York, NY, US: Guilford Press; 2015. p. 455-74.

10. Lipowska M. Dysleksja i ADHD - współwystępujące zaburzenia rozwoju. Neuropsychologiczna analiza deficytów pamięci [Dyslexia and ADHD - comorbidity of developmental disorders. The neuropsychological analysis of memory deficits]. Warszawa: Scholar; 2011.

11. Brandt J. Diet in Brain Health and Neurological Disorders: Risk Factors and Treatments. Brain Sciences. 2019;9(9):234.

12. Rutter TM, Arnett AB. Temperament Traits Mark Liability for Coexisting Psychiatric Symptoms in Children With Elevated ADHD Symptoms. Journal of Attention Disorders. 2020:1087054720943282.

13. Krakowski AD, Cost KT, Anagnostou E, Lai M-C, Crosbie J, Schachar R, et al. Inattention and hyperactive/impulsive component scores do not differentiate between autism spectrum disorder and attention-deficit/hyperactivity disorder in a clinical sample. Molecular Autism. 2020;11(1):28.

14. Barnow S, Schuckit M, Smith T, Spitzer C, Freyberger HJ. Attention problems among children with a positive family history of alcohol abuse or dependence and controls. Prevalence and course for the period from preteen to early teen years. European addiction research. 2007;13(1):1-5.

15. Bouchard G, Saint-Aubin J. Attention deficits and divorce. Can J Psychiatry. 2014;59(9):480-6.

16. Roberts SD, McDonald KP, Danguecan A, Crosbie J, Westmacott R, Andrade B, et al. Longitudinal Academic Outcomes of Children with Secondary Attention Deficit/Hyperactivity Disorder following Pediatric Stroke. Developmental Neuropsychology. 2019;44(4):368-84.

17. Vázquez-Marrufo M, García-Valdecasas Colell M, Galvao-Carmona A, Sarrias-Arrabal E, TirapuUstárroz J. El Attention Network Test en el estudio de los déficits cognitivos de pacientes con trastorno por déficit de atención. [The Attention Network Test in the study of cognitive impairment of ADHD patients]. Rev Neurol. 2019;69(10):423-32. 
18. Posner MI, Petersen SE. The attention system of the human brain. Annu Rev Neurosci. 1990;13:25-42.

19. Fan J, McCandliss BD, Sommer T, Raz A, Posner MI. Testing the Efficiency and Independence of Attentional Networks. Journal of Cognitive Neuroscience. 2002;14(3):340-7.

20. Rueda MR, Fan J, McCandliss BD, Halparin JD, Gruber DB, Lercari LP, et al. Development of attentional networks in childhood. Neuropsychologia. 2004;42(8):1029-40.

21. Greene DJ, Barnea A, Herzberg K, Rassis A, Neta M, Raz A, et al. Measuring attention in the hemispheres: the lateralized attention network test (LANT). Brain and cognition. 2008;66(1):21-31.

22. Fan J, Gu X, Guise KG, Liu X, Fossella J, Wang H, et al. Testing the behavioral interaction and integration of attentional networks. Brain and cognition. 2009;70(2):209-20.

23. Rueda MR, Fan J, McCandliss BD, Halparin JD, Gruber DB, Lercari LP, et al. Development of attentional networks in childhood. Neuropsychologia. 2004;42(8):1029-40.

24. Callejas A, Lupiáñez J, Tudela P. The three attentional networks: on their independence and interactions. Brain and cognition. 2004;54(3):225-7.

25. Roca J, Castro C, López-Ramón MF, Lupiáñez J. Measuring vigilance while assessing the functioning of the three attentional networks: The ANTI-Vigilance task. Journal of Neuroscience Methods. 2011;198(2):312-24.

26. Arora S, Lawrence MA, Klein RM. The Attention Network Test Database: ADHD and CrossCultural Applications. Frontiers in Psychology. 2020;11(388).

27. Liu L, Johnson HL, Cousens S, Perin J, Scott S, Lawn JE, et al. Global, regional, and national causes of child mortality: an updated systematic analysis for 2010 with time trends since 2000. Lancet. 2012;379(9832):2151-61.

28. Shamseer L, Moher D, Clarke M, Ghersi D, Liberati A, Petticrew M, et al. Preferred reporting items for systematic review and meta-analysis protocols (PRISMA-P) 2015: elaboration and explanation. BMJ : British Medical Journal. 2015;349:g7647.

29. WHO. International classification of diseases for mortality and morbidity statistics (11th Revision): Retrieved from https://icd.who.int/browse11/I-m/en; 2019.

30. Aswathi A, Rajendiren S, Nimesh A, Philip RR, Kattimani S, Jayalakshmi D, et al. High serum testosterone levels during postpartum period are associated with postpartum depression. Asian Journal of Psychiatry. 2015;17:85-8. 
31. Eapen BR. EndNote 7.0. Indian journal of dermatology, venereology and leprology. 2006;72(2):165-6.

32. Page MJ, McKenzie JE, Bossuyt PM, Boutron I, Hoffmann TC, Mulrow CD, et al. The PRISMA 2020 statement: an updated guideline for reporting systematic reviews. BMJ. 2021;372:n71 .

33. Sterne JAC, Savović J, Page MJ, Elbers RG, Blencowe NS, Boutron I, et al. RoB 2: a revised tool for assessing risk of bias in randomised trials. BMJ. 2019;366:14898.

34. Sterne JA, Hernán MA, Reeves BC, Savović J, Berkman ND, Viswanathan M, et al. ROBINS-l: a tool for assessing risk of bias in non-randomised studies of interventions. BMJ. 2016;355:i4919.

35. Ettenberger M, Odell-Miller H, Rojas Cárdenas C, Torres Serrano S, Parker M, Camargo Llanos SM. Music Therapy With Premature Infants and Their Caregivers in Colombia - A Mixed Methods Pilot Study Including a Randomized Trial. Voices: A World Forum for Music Therapy, [SI]. 2014;14(2).

36. Wells GA, Shea B, O'connell D, Peterson J, Welch W, Losos M, et al. The Newcastle-Ottawa Scale (NOS) for Assessing the Quality if Nonrandomized Studies in Meta-Analyses. ScienceOpen. 2015.

37. Higgins JPT, Thomas J, Chandler J, Cumpston M, Li T, Page MJ, et al. Cochrane Handbook for Systematic Reviews of Interventions: Wiley; 2019.

38. Team RC. R: A language and environment for statistical computing. 2013.

\section{Figures}

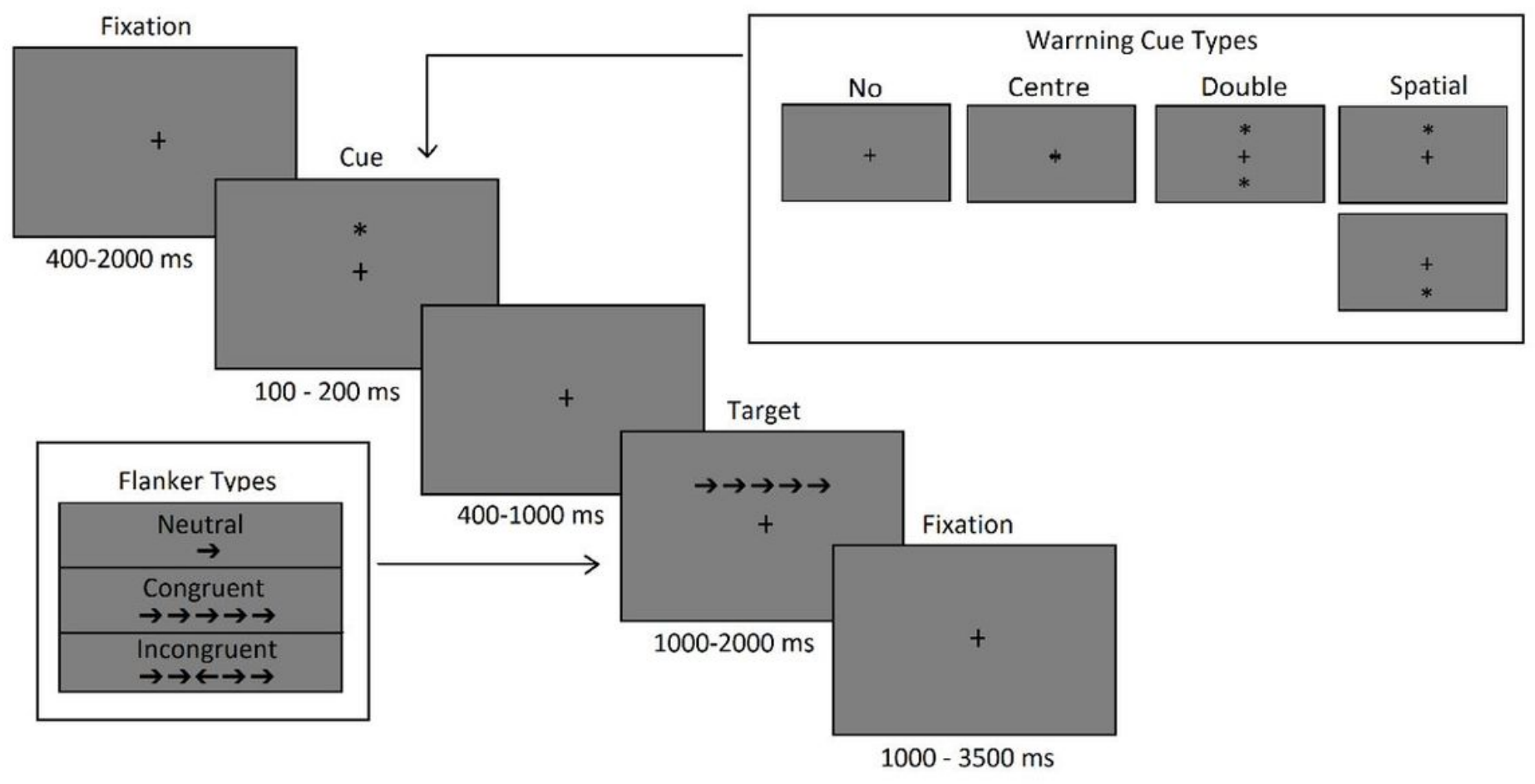




\section{Figure 1}

Attention Network Task Protocol - Made based on Original ANT version (Fan, et al.,2002) and another previous research (Rueda, et al., 2003; Vázquez-Marrufo et al., 2019)

\section{Supplementary Files}

This is a list of supplementary files associated with this preprint. Click to download.

- Additionalfile1.docx 